\title{
Opportunistic Wireless Access Networks
}

\author{
(Invited Paper)
}

\author{
Kaustubh S. Phanse \\ Luleå University of Technology \\ Department of Computer Science and Electrical \\ Engineering \\ 97187 Luleå, Sweden \\ +46 920-49-1428 \\ kphanse@Itu.se
}

\author{
Johan Nykvist \\ Luleå University of Technology \\ Department of Computer Science and Electrical \\ Engineering \\ 97187 Luleå, Sweden \\ +46 920-49-2898 \\ johan@csee.Itu.se
}

\begin{abstract}
Today, intermittent connectivity is common in wireless access networks and it seems like mobile users will have to deal with discontinuous network access in the future. But existing network protocol architectures are not resilient to disruption of communication links and often communication fails when faced with sporadic connectivity. We discuss a new paradigm for wireless access called opportunistic collaborative networking, where communication is disruption-tolerant and wireless devices exploit any opportunity of communication, albeit temporary, to forward messages towards the destination. For developing protocols for these innovative networks requires understanding of their structure and dynamics. We present some results from analysis of real-life traces representing two such mobile network environments with ad-hoc, intermittent connectivity.
\end{abstract}

\section{Categories and Subject Descriptors}

D.3.3 [Network architecture and Design]: Wireless communications

\section{General Terms}

Algorithms, Design, Performance

\section{Keywords}

Opportunistic, Collaborative networks, connectivity, trace, model.

\section{INTRODUCTION}

With the proliferation of a variety of wireless access technologies, seamless connectivity and anywhere, anytime computing are commonly touted as the paradigms for serving mobile users. Further, broadband wireless access is described as the panacea for the last-mile problem. While the vision of seamless connectivity and broadband wireless Internet access is attractive, it is far from reality. For various regulatory, technical and economical reasons, wireless access networks worldwide fail to fulfill the promise of

Permission to make digital or hard copies of all or part of this work for personal or classroom use is granted without fee provided that copies are not made or distributed for profit or commercial advantage and that copies bear this notice and the full citation on the first page. To copy otherwise, or republish, to post on servers or to redistribute to lists, requires prior specific permission and/or a fee.

AccessNets'06, September 4-6, 2006, Athens, Greece.

Copyright 2006 ACM 1-59593-513-4...\$5.00. continuous, high-bandwidth, and affordable service.

Cellular networks (e.g., GSM/UMTS) are the most common option for mobile wide-area network access. Their coverage continues to be variable and intermittent [1]. In terms of performance, 2/2.5G networks provide low bandwidth access. While $3 \mathrm{G}$ promises high bandwidth access, it is expensive and its metered service is not viewed as a true option for extensive Internet access. The potential success of newer technologies using licensed spectrum such as IEEE 802.16 (WiMax) remains questionable. The substantial investment made in $3 \mathrm{G}$ licenses and infrastructure is a deterrent for network operators to adopt a new technology for mobile broadband access. As a broadband solution to the last-mile problem in poor and developing countries and in rural and remote areas, WiMax and other licensed wireless access technologies face the chicken-and-egg problem of the simultaneous need for both a market and an infrastructure. Providing continuous broadband coverage in rural areas can be an expensive endeavor for network operators due to the sparse population density, e.g., [2], challenging terrain, and lack of other relevant infrastructure such as reliable supply of electricity [3]. IEEE 802.11 (WiFi) has experienced widespread proliferation thanks to its operation in the unlicensed spectrum and cheap hardware. But coverage of WiFi hotspots is limited to few hundred meters.

In spite of efforts to extend the coverage of infrastructure wireless networks, for instance, using the multi-hop ad-hoc and mesh networking approach [4], intermittent connectivity prevails. Still, wireless access networks today are architected for providing continuous, synchronous access to users; to a great extent this can be attributed to the end-to-end communication paradigm prevalent in the Internet. Irrespective of the kind of network services a user is interested in, the end-user is expected to be physically present within the coverage of these infrastructure based access networks for any communication to take place. This, we believe, is a major hurdle for extending network access to a sizeable user population who cannot afford to be physically present within the coverage area of the nearest base station or hotspot [3] and to mobile users who find it cumbersome keeping track of their intermittent network access as they move in and out of the sporadic coverage. While continuous, connectivity is essential for synchronous applications such as real-time video and voice conferencing, there are many asynchronous applications: cached Web access, electronic mail, multimedia messaging, newscasting, file sharing, and blogging, to name a few that do not need continuous network access. But today's networks and protocols are not resilient to 
disruption of communication links, and are not designed to exploit intermittent availability of network resources.

In this paper, we consider an emerging paradigm of opportunistic collaborative networking that overcomes these hurdles and supports a more practical whenever, wherever style of computing. Moving away from the traditional centralized operational model of access networks, we envision decentralized, self-organizing, opportunistic access networks in the future, those that incorporate intermittent connectivity and support natural human communication and usage patterns. Many challenges arise in implementing this new class of networks, e.g., handling heterogeneous types of devices with different communication characteristics, efficient routing in presence of network partitioning, disruption-tolerant transport layer and a connectivity-aware application design. Understanding the nature and structure of these complex and dynamic networks is crucial for engineering technical solutions as well as architecting business models to commercialize these networks in the future. In our ongoing work, we attempt to model the behavior of intermittently connected mobile access networks using real-life traces.

The rest of the paper is organized as follows. In Section 2, we define opportunistic, collaborative access networks. In Section 3, we analyze real-life wireless connection traces of two different mobile network environments and derive the emergent statistical structure. We present our concluding remarks in Section 4.

\section{OPPORTUNISTIC COLLABORATIVE NETWORKS}

Many mobile and nomadic computing scenarios exist and new applications are emerging where users would like to use asynchronous and "real-enough time" services that exploit any opportunity of communication, albeit temporary, with other wireless devices or infrastructure in the vicinity. These contact opportunities could be periodic, coordinated, spontaneous or a combination therein. Concrete examples are: community mobile networks such as DakNet [3] and Sámi Network Connectivity [5] in rural and underserved regions; emergency and disaster relief operations where sporadic connectivity is what the network can support at best, vehicular ad-hoc networks [6] and ad-hoc peer-topeer networks [7].

As shown in Figure 1, these networks typically comprise heterogeneous wireless devices in motion (e.g., portable devices carried by mobile users, vehicles, high-altitude platforms) that form dynamic clusters or islands of connectivity, cooperate in routing data and take advantage of infrastructure network resources as and when they become available. It may take multiple discontinuous wireless contacts or hops before the data is carried from the source to the destination over a period of time. For instance, the dotted circles in Figure 1 illustrate dissemination of data from node 4 to node 2 over a period of time without contemporaneous end-to-end connectivity.

These opportunistic collaborative networks relax the "always-on" assumption and allow data transport even in the absence of contemporaneous end-to-end path between the source and destination. This paradigm is a radical departure from the traditional end-to-end communication model pursued in the Internet, and falls in the general space of delay- and disruption- tolerant networking (DTN) [8, 9]. The DTN concept was incubated primarily in the InterPlanetary Internet (IPN) Project, a special interest group within the Internet Society. The motivation was the need for a new architecture that could address internetworking of and interoperability between significantly disparate network environments envisioned in an interplanetary Internet [10]. These environments would be characterized by a wide variety of constraints and extreme conditions such as long, variable delays (minutes, hours or days) and frequent disruption of links, unlike the existing Internet we know. Since then the DTN idea has been found relevant and useful for many terrestrial applications, e.g., [3, 5, 6, 7] and has attracted considerable attention within the research community in the last few years.

Opportunistic collaborative networks have the potential of enabling new kinds of services that are capable of utilizing resources as and when they are available. This allows the network functioning to be versatile and driven by the natural usage needs rather than the technological aspects. We believe that this is the raison d'être that makes opportunistic collaborative networking powerful and attractive.

Let us now elaborate on the "opportunistic" and "collaborative" characteristics to understand what types of behavior and functionality could comprise these networks.

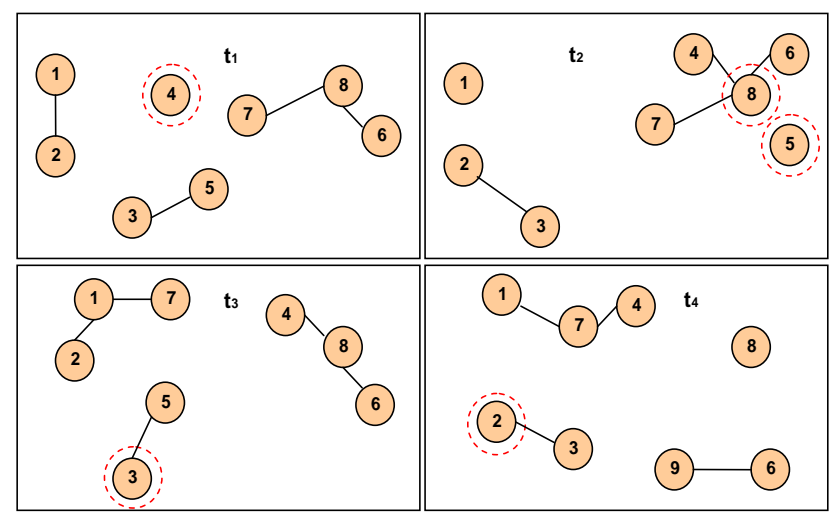

Figure 1. Data routing using opportunistic contacts

\subsection{Opportunistic networking}

The literal meaning of the term "opportunistic" is evident-the tendency of network devices to exploit available resources in the network as and when possible. In the context of communication networks, though, it represents many more subtle properties. Opportunistic networks are intrinsically fault tolerant for they are not limited by the end-to-end connectivity assumption. These networks are distributed and self-organizing in that the control and management is largely up to the individual devices or users (within the boundaries defined by the network operator's policies, if part of a commercial network). The communication in these networks is localized, i.e., decisions such as routing are made by devices based on locally available information. Opportunistic also means being able to take advantage of locally accessed global information, where devices implicitly convey global reachability information strictly through local interaction. 
Communication opportunities in a network can arise in different forms. They can be:

1. Deterministic-periodic connectivity, e.g., in an interplanetary network based on the movement patterns of planets and satellites, or connectivity that is a function of time synchronization among sensors;

2. Coordinated - a group of users deciding to meet at a particular location at a certain time to share data;

3. Spontaneous - when two or more devices meet by chance, e.g., two or more users with common interests meeting at an airport.

Various forms of user interactions together may lead to complex probabilistic connectivity patterns that, for instance, closely follow the social behavioral patterns of users. On a finer temporal granularity, the interconnectivity among devices may look spontaneous. Though from a bird's eye view, it exhibits an emergent behavior where the collective conduct of devices imparts interesting spatio-temporal statistical properties for the network as a whole, e.g., distribution of inter-contact times and contact times, clustering.

\subsection{Collaborative networking}

The term collaborative, in the context of communication networks, represents the various ways in which users and devices cooperate to mutually benefit from one another's role in the network, to maximize the network's utility and possibly to attain a common goal. There are several examples where the collaborative nature transpires as an integrated feature of the network. For instance, peer-to-peer file sharing applications such as BitTorrent [11] have incentive for cooperation built into the design; sensors in a wireless sensor network may implement energy-efficiency algorithms to attain the goal of network longevity; certain networks are deployed with a specific mission requiring users and devices to cooperate actively, e.g., emergency and rescue operations and NASA's interplanetary network. Alternatively, in a more anarchical setting, altruism on the part of certain users or the use of appropriate incentives, for instance, based on pricing mechanisms [12] or using game theoretic models [13] could trigger cooperation.

\section{MODELING MOBILE NETWORKS WITH AD HOC CONNECTIVITY}

The connection patterns of wireless mobile devices - how long and how frequently they connect - dictate the ability to route and transport data in these networks, in turn determining the performance limits of deployed services (e.g., end-to-end delay, packet delivery ratio). Until now, it has been a common practice in the mobile ad-hoc network community to employ synthetic spatial mobility models in order to implicitly generate temporal connection patterns. The mobility models generally focus on parameters such as initial distribution of nodes, maximum velocity and pause time of nodes; they often hide some of the crucial behavioral properties of the network that affect the performance and may inadvertently impair the accuracy of the results and conclusions. A prime example is the previously popular Random Way Point mobility model [14].

We argue that temporal connection models are better suited than spatial mobility models - instead of creating temporal connection patterns indirectly via spatial mobility models, we advocate using temporal connection models directly. This approach has at least two distinct advantages: first, it is easier to model realistic connection patterns, e.g., based on real-life traces, than mobility patterns; second, we are able to understand the network behavior much better, which facilitates development of practical protocols for these network environments.

To this end, we have developed methods to analyze real-life connection traces, identify significant stochastic properties, and create realistic connection models for simulation and formal analysis. We analyze traces from the MIT Reality Mining project [15] (specifically, a Bluetooth connection trace between mobile phones), henceforth referred to as Reality, and the Haggle project [16], henceforth referred to as Haggle. The traces capture behavior of two different intermittently connected mobile ad-hoc human social networks: the users in the Reality trace are students at MIT carrying Bluetooth-enabled smartphones, while those in the Haggle trace are attendees at the IEEE conference INFOCOM 2005 carrying Bluetooth enabled iMote sensors. Here, we present some results from our on-going trace analysis.

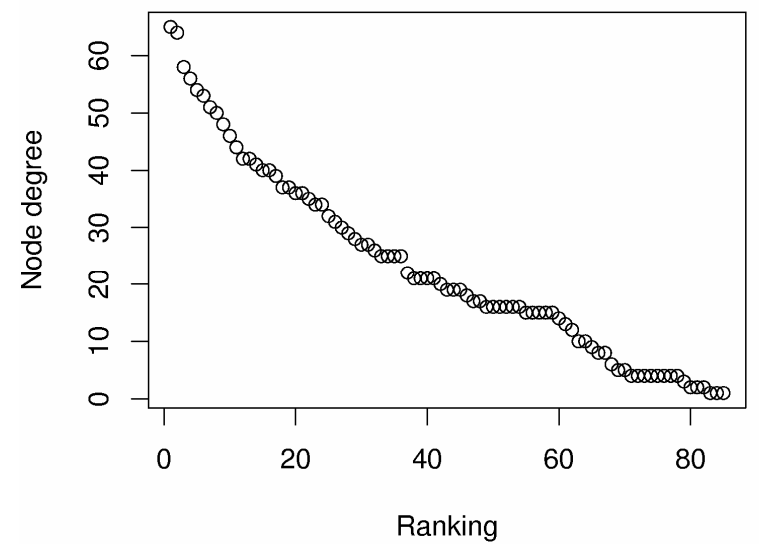

(a) Reality trace

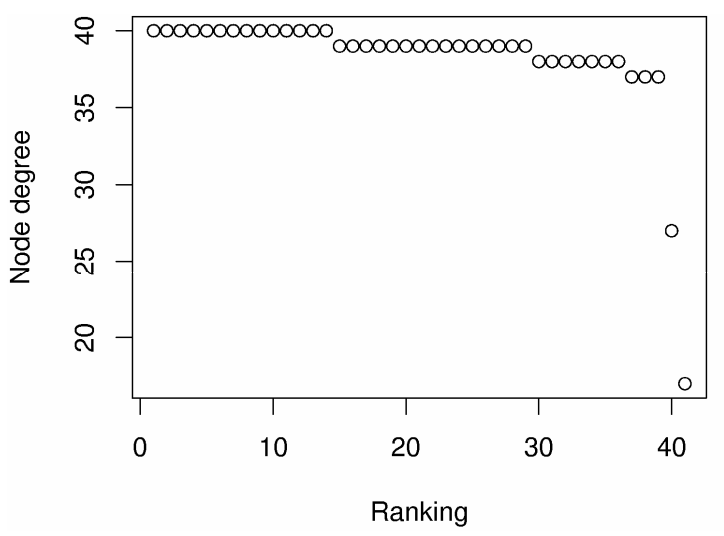

(b) Haggle trace

Figure 2. Node degree distribution

To derive emergent topological properties, we create an Aggregated Connection Graph (ACG), which is a weighted graph where the nodes represent mobile entities and the edges represent connections between the mobile entities. The edge weights 
represent the cumulative connection time between the mobile entities. The diagrams in Figures 2(a) and 2(b) show the node degree distributions for Reality and Haggle. The degree distribution of the Reality nodes is approximately linear. In contrast, almost all Haggle nodes have degrees of approximately 40. The large difference in the degree distributions is attributed to how people carrying the nodes associate in the different environments.

The log-log diagrams in Figures 2(a) and 2(b) show the edge weight (node-pair total connection time) distributions for Reality and Haggle. Both distributions are heavy-tailed, a few node pairs are connected several magnitude of orders longer than the larger part of the node pairs. We rank the edges according to their weights such that the highest ranked edge has the longest total connection time.

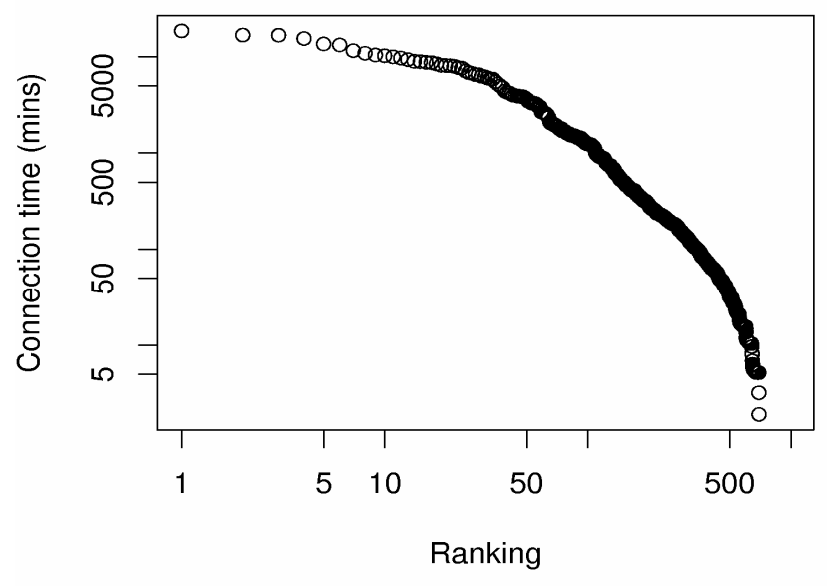

(a) Reality trace

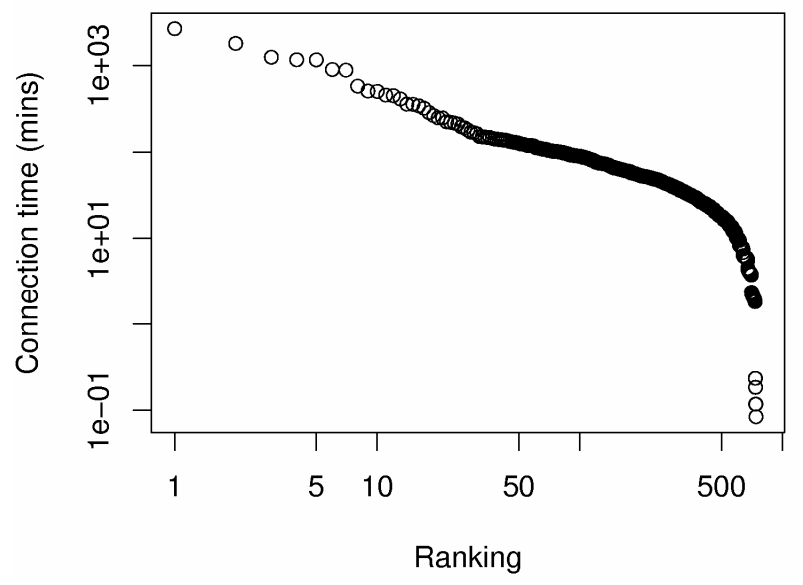

(b) Haggle trace

Figure 3. Total connection time distribution

To further investigate the relation between topology and edge weights, we define a network's backbone as a sub-graph of the ACG that is induced by the nodes that are connected by the top $5 \%$ ranked edges. Figure 4 shows the occurrence of backbones. The Reality backbone consists of 28 nodes (approximately $28 \%$ of the total number of nodes) and 50 edges. The thickness of the edges indicates the aggregate connection time. The Reality backbone is more clustered than the Haggle backbone. We also observed communities - groups of devices internetworking more frequently. In Figure 5, we show communities in the Reality trace; the top $5 \%$ ranked edges per node have been plotted. The thick edges represent the backbone links.

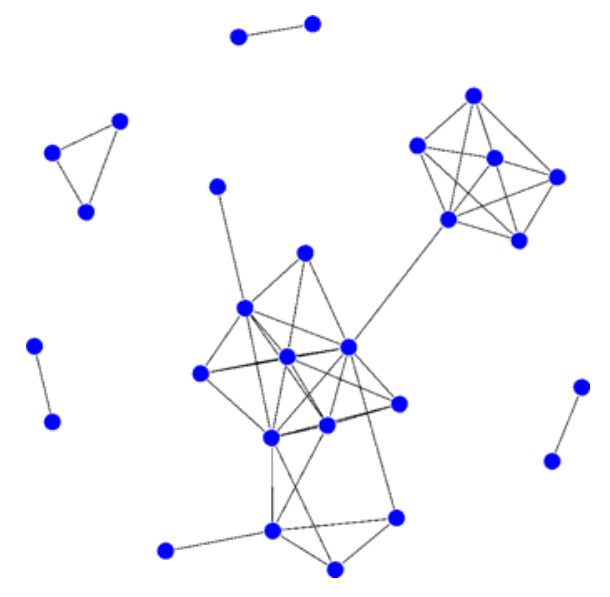

(a) Reality trace

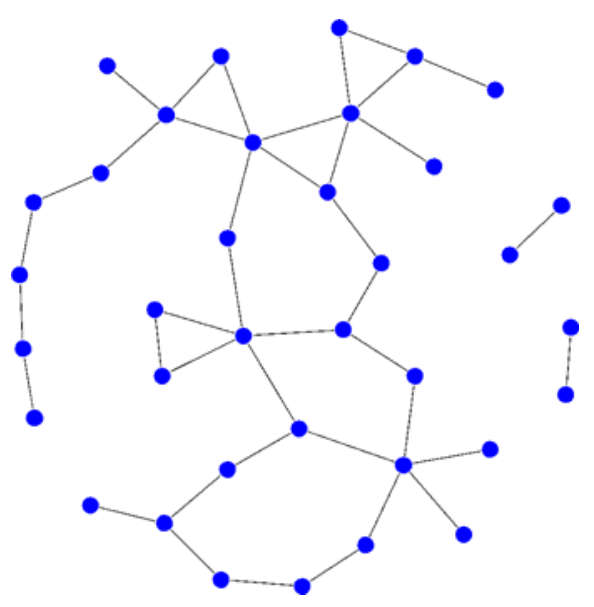

(b) Haggle trace

Figure 4. Occurrence of backbone

\section{DISCUSSION}

Incorporating opportunistic and collaborative features into wireless access networks can greatly enhance the utility of existing access networks presenting unforeseen opportunities for technological and entrepreneurial innovations. These networks will evolve around social behavior and usage patterns of mobile users and hence will be complex in nature. Understanding the dynamics of these networks is crucial for engineering practical solutions. Our preliminary analysis of real-life wireless connection traces has brought out some interesting statistical and topological properties of real-life mobile human social networks. 
We are currently developing a software tool to help simulate and visualize network behavior. Our long term goal is to propose analytical and simulation models to facilitate in-depth study of such complex networks. In addition to the emergent behavior, in the future, we hope to capture granular dynamic properties that will aid in designing data routing and forwarding strategies, feasible content distribution services, and deployment of infrastructure such as base stations and data storage facilities at key locations.

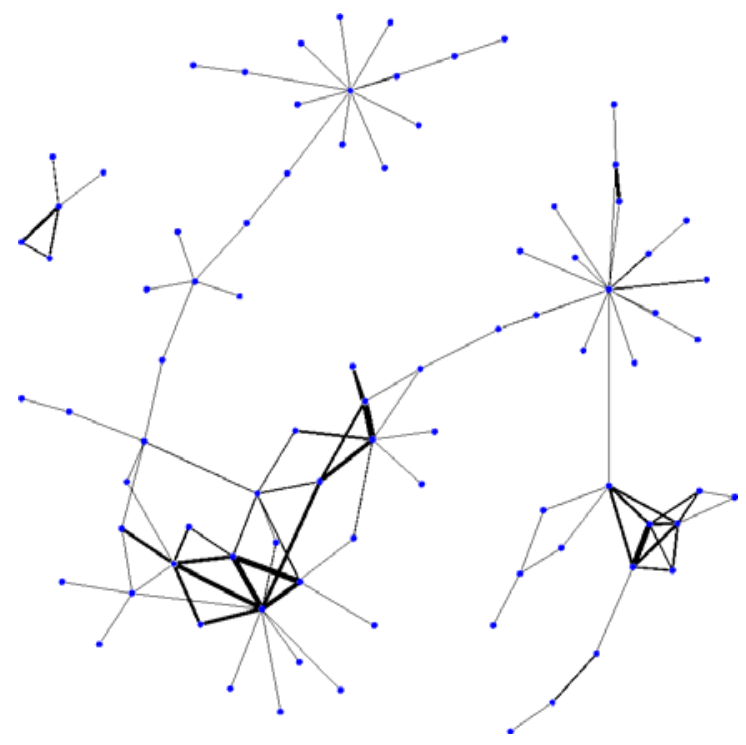

Figure 5. Occurrence of communities (Reality trace)

\section{REFERENCES}

[1] Worldwide GSM coverage information. http://www.gsmworld.com/roaming/gsminfo/index.shtml.

[2] C. Demunter, “The digital divide in Europe,” Report released by Eurostat, the Statistical Office of the European Communities, October 2005. www.eu.int/comm/eurostat/.

[3] A. Pentlund, R. Fletcher and A. Hasson, "DakNet: Rethinking Connectivity in Developing Nations," IEEE Computer, pp. 78-83, vol. 37, no. 1, January 2004.
[4] R. Bruno, M. Conti and E. Gregori, "Mesh networks: commodity multihop ad hoc networks," IEEE Communications Magazine, pp. 123-131. vol. 43, no. 3, March 2005.

[5] Sámi Network Connectivity project. http://www.snc.sapmi.net/

[6] J. LeBrun, C.-N. Chuah and D. Ghosal, "Knowledge Based Opportunistic Forwarding in Vehicular Wireless Ad Hoc Networks," Proceedings of IEEE Vehicular Technology Conference (VTC), pp. 1-5, 2005.

[7] P. Hui, A. Chaintreau, J. Scott, R. Gass, J. Crowcroft and C. Diot, "Pocket Switched Networks and human Mobility in Conference Environments," Proceedings of the ACM SIGCOMM Workshop on Delay-Tolerant Networking (WDTN), August 2005.

[8] IRTF Delay-Tolerant Networking Research Group (DTNRG). http://www.dtnrg.org

[9] DARPA Disruption Tolerant Networks. http://www.darpa.mil/ato/programs/DTN/

[10] S. Burleigh et al., "Delay-Tolerant Networking: An Approach to Interplanetary Internet," IEEE Communications Magazine, pp. 128-136, vol. 41, no. 6, June 2003.

[11] BitTorrent. http://www.bittorrent.com/

[12] J. Crowcroft, R. Gibbens, F. Kelly, and S. Östring, "Modelling Incentives for Collaboration in Mobile Ad Hoc Networks," Proceedings of the $1^{\text {st }}$ Workshop on Modeling and Optimization in Mobile, Ad Hoc and Wireless Networks, March 2003.

[13] V. Srivastava, "Node Cooperation in Ad Hoc Networks - A Game-Theoretic Approach,” Ph.D. preliminary examination report, Department of Computer Science and Electrical Engineering, Virginia Tech, May 2006.

[14] J. Yoon, M. Liu and B. Noble, "Random Waypoint Considered Harmful," Proceedings of IEEE INFOCOM, 2003.

[15] MIT Reality Mining project. http://reality.media.mit.edu/

[16] Haggle project. http://www.haggleproject.org/ 